\title{
A mixed methods exploration of families' experiences of the diagnosis of childhood spinal muscular atrophy
}

\begin{abstract}
Sally Lawton ${ }^{1}$, Chriselle Hickerton ${ }^{2}$, Alison D Archibald ${ }^{2}$, Belinda J McClaren ${ }^{1,3}$ and Sylvia A Metcalfe ${ }^{\star, 1,3}$
Spinal muscular atrophy (SMA) is an autosomal recessive neuromuscular disease with a carrier frequency of 1 in 41 in Australia. Childhood SMA is classified into three types based on the age at which children present with symptoms and the clinical severity. Families' experiences leading up to the diagnosis have not been described, but are important when considering the potential for a diagnostic odyssey. Using a mixed methods approach, data were collected from interviews and a national survey of families of children with SMA to explore their experiences of this journey. The combined findings $(n=28)$ revealed that the journey to receiving a diagnosis was protracted. The time from first noticing symptoms to finally receiving a diagnosis was emotional and frustrating. Once parents or other family members became aware of symptoms, almost all had consulted with multiple different health professionals before the diagnosis was ultimately made. Not surprisingly, receiving the diagnosis was devastating to the families. The nature of the information and the way it was given to them was not always optimal, particularly because of the difficulties predicting clinical severity. Most felt that their child could have been diagnosed earlier and, although there were mixed views around the benefit of this for their child, they felt it may have reduced the emotional impact on families. Overall, families were more in favour of population carrier screening for SMA when compared with newborn screening of the population. Despite an increasing awareness of SMA, the diagnostic delay continues to have negative impacts on families.
\end{abstract}

European Journal of Human Genetics (2015) 23, 575-580; doi:10.1038/ejhg.2014.147; published online 30 July 2014

\section{INTRODUCTION}

Developmental delay is apparent when children display a rate of development that is slower in one or more areas than most children of the same age. ${ }^{1}$ As there is wide variation in the ages at which children reach their developmental milestones, it is difficult to pinpoint the most appropriate time to initiate clinical investigations into the cause of the suspected delay. Therefore, health professionals face many challenges in detecting and diagnosing developmental delay. At least $20 \%$ of cases of developmental delay have known genetic causes, for example, chromosomal abnormalities and single gene disorders, such as fragile X syndrome (FXS), Duchenne muscular dystrophy (DMD) and other dystrophies such as spinal muscular atrophy (SMA). In the case of $\mathrm{FXS}^{2}$ and DMD, ${ }^{3,4}$ there is evidence that parents may undergo a protracted journey before a diagnosis is made, with associated negative impacts on the families and lost opportunities for earlier intervention and reproductive decision-making.

It is likely that the situation is similar for other inherited conditions for which symptoms are not obvious in the early neonatal period, such as some types of SMA. However, there is limited research around parental experiences of receiving a diagnosis of SMA. SMA is an autosomal recessive neuromuscular disease with a carrier frequency of 1 in 41 reported in the Australian population ${ }^{5}$ and an estimated incidence of 1 in 6000 to 1 in 10000 live births internationally. ${ }^{6,7}$ Degeneration of motor neurons in the spine and lower brainstem lead to muscle weakness and atrophy. ${ }^{8}$ This degeneration has been shown to be due to homozygous defects of the spinal motor neuron gene,
SMN1. ${ }^{9,10}$ The presence of varying numbers of copies of the homologous SMN2 gene influence the clinical severity of the condition. ${ }^{11}$ Childhood SMA is classified into three types (type 1, type 2 and type 3 ) based on age of onset and disease severity. ${ }^{12}$ SMA type 1, the most severe form, presents within the first 6 months of life and death typically occurs before 2 years of age. ${ }^{12}$ The onset of symptoms for SMA type 2 occurs between 6 and 18 months of age; children are not able to walk independently; survival past 2 years of age is common; historically, children rarely survived into adulthood, although this has changed in recent years with management. ${ }^{12,13}$ SMA type 3 is the mildest form of childhood SMA with age of onset greater than 18 months, and is further sub-classified into types $3 \mathrm{a}$ and $3 \mathrm{~b}$, when presentation is before or after 3 years of age, respectively; children are able to walk, but many later lose ambulation, and they have problems with gross and fine motor skills; they achieve a reasonably normal lifespan. ${ }^{13}$ Advances in respiratory care have improved survival rates in individuals with SMA types 2 and $3 .{ }^{13}$

Recently, Farrar et $a^{13}$ described the detailed natural history and survival probabilities of 70 patients presenting to a specialised clinic in Australia between 1995 and 2010. These patients had undergone genetic testing following initial clinical diagnostic investigations after parental or health professional concern about development, primarily symptoms such as hypotonia, weakness and motor delay. Although these authors briefly mentioned parental reports of the early clinical symptoms, no studies have described parental experiences of leading up to and receiving a diagnosis, which could indicate a potential diagnostic odyssey, and the impact on families. ${ }^{13}$

\footnotetext{
${ }^{1}$ Murdoch Childrens Research Institute, Department of Paediatrics, The University of Melbourne, Parkville, Australia; ${ }^{2}$ GDipGenetCouns, Murdoch Childrens Research Institute, Victorian Clinical Genetics Services, Department of Paediatrics, The University of Melbourne, Parkville, Australia

*Correspondence: Professor SA Metcalfe, Murdoch Childrens Research Institute, Royal Children's Hospital, Flemington Rd, Parkville, VIC 3052 , Australia. Tel: +61 3 8341 6309, Fax: +61 38341 6212; E-mail: sylvia.metcalfe@mcri.edu.au

${ }^{3}$ Co-senior authors.

Received 22 April 2014; revised 10 June 2014; accepted 25 June 2014; published online 30 July 2014
} 
This study aimed to explore the experiences of family members regarding the journey to receiving a diagnosis, and their views on the potential for earlier diagnosis through screening.

\section{MATERIALS AND METHODS}

This study used a mixed methods approach collecting both qualitative and quantitative data through interviews and a survey. The approach used a triangulation design in which the interpretation of the findings was based on mixing the results from both types of data. ${ }^{14}$ This study was approved by the Human Research Ethics Committee of The Royal Children's Hospital (RCH), Melbourne, Victoria, Australia (HREC 31047C).

\section{Recruitment and sampling}

Parents and relatives of children with SMA types 2 and 3 were purposively sampled into the qualitative interview component of the study focused on the journey to diagnosis, as it was assumed they would be more likely to have received a diagnosis beyond the first few months of the child's life compared with parents and relatives of children diagnosed with SMA type 1. Participants were recruited through advertising the study with the Spinal Muscular Atrophy Association of Australia (SMAAA), via the Association's regular email newsletter. People interested in participating contacted the research team directly. Snowball sampling, when a participant gives the study information to another eligible person, ${ }^{15}$ was also carried out; therefore, interviewed parents were asked to mention the study to other family members who might want to participate. Eligibility criteria for interviews were: $\geq 18$ years of age; able to speak, read and write English; parents, siblings or second-degree relatives of people with SMA types 2 or 3 .

A link to an online survey was emailed by the SMAAA to all members and hard copies were mailed upon request. Eligible survey participants were: $\geq 18$ years of age; able to speak, read and write English; parents, siblings or second-degree relatives of people with SMA types 1,2 or 3 . SMA type 1 was also included, as this survey was intended to explore broader information about families' views on population genetic screening.

\section{Data collection and analysis}

Semi-structured interviews were chosen initially to capture the breadth and depth of participants' views. We used a flexible question guide informed by previous literature and discussion with clinicians at RCH. Open-ended questions and follow-up prompts encouraged exploration of experiences with SMA in the family, including: symptoms that were first noticed; the process of trying to find out the cause of the problem; receiving a diagnosis and its impact on the family; perceptions of the potential for an earlier diagnosis; and views on genetic screening. Interviews were conducted either at the RCH or by telephone, and were audio-recorded, transcribed verbatim and de-identified, with participants assigned a number. Initially, transcripts were read a number of times, allowing the authors to become familiar with the data. Transcripts were imported into NVivo 2009 software (QSR International, Doncaster, VIC, Australia) to manage systematic coding. Data were analysed using inductive content analysis, in which broad categories were initially developed based on concepts emerging from the data. ${ }^{16} \mathrm{~A}$ constant comparative approach was used to examine similarities and differences within these categories, which were explored further to identify themes. ${ }^{17}$ Transcripts were coded independently by at least two researchers for rigour, and themes were discussed by the research team to achieve consensus.

A survey was developed based on data emerging from the interviews. The survey included closed and open-ended questions on participant demographics, initial symptoms of SMA noticed by parents or health professionals, contact with health professionals, how a diagnosis was obtained, and views on changes to the way SMA may be identified and diagnosed in the future. The researchers developed and refined the questions in an iterative process. The survey was piloted with the interviewed participants to check for clarity and ease of response to the questions and then made available online with invitations through SMAAA. Data were downloaded into Excel and openended responses were coded according to the categories developed from the interviews. The data from the interviews and survey were mixed for interpretation. ${ }^{14}$

\section{RESULTS}

\section{Description of participants}

Interviews were conducted in 2011 with eight participants residing in three different states in Australia: five mothers of a child with type 2 (P1-3, 5-6); one mother of two children, each with type 3 (P4); one grandmother of a child with type 2 (P21); and one sister of an adult with type 2 (P20). A further 20 surveys were received in 2012. Participant characteristics $(n=28$, representing 25 families) are shown in Table 1 ; the majority $(n=17)$ were mothers of a child with SMA. Sixteen respondents to the survey were parents (seven parents of children with SMA type 1, six of type 2 and three

\section{Table 1 Participant characteristics}

\begin{tabular}{|c|c|}
\hline Characteristic & n (and participant code) \\
\hline Relationship to child & $(\mathrm{n}=28)$ \\
\hline Mother & $17(\mathrm{P} 1-17)$ \\
\hline Father & 2 (P18-19) \\
\hline Siblinga & 1 (P20) \\
\hline Grandmother ${ }^{b}$ & 3 (P21-23) \\
\hline Uncle/Aunt ${ }^{\mathrm{C}}$ & 4 (P24-27) \\
\hline Other ${ }^{\mathrm{d}}$ & $1(\mathrm{P} 28)$ \\
\hline Relationship status & $(\mathrm{n}=28)$ \\
\hline Married & 18 \\
\hline Divorced & 2 \\
\hline Separated & 1 \\
\hline de facto & 4 \\
\hline Single & 1 \\
\hline Partner, not living together & 2 \\
\hline Age range & $(\mathrm{n}=28)$ \\
\hline $21-30$ years & 2 \\
\hline $31-40$ years & 16 \\
\hline $41-50$ years & 7 \\
\hline $51-60$ years & 1 \\
\hline $61-70$ years & 2 \\
\hline Occupation & $(n=25)^{e}$ \\
\hline Professional & 8 \\
\hline Home duties & 9 \\
\hline Carer & 4 \\
\hline Trade & 1 \\
\hline Manager or administration & 3 \\
\hline Highest level of education attained & $(\mathrm{n}=25)^{\mathrm{e}}$ \\
\hline Year 11 or below & 5 \\
\hline Year 12 or equivalent & 2 \\
\hline Tertiary certificate/diploma & 9 \\
\hline Trade/apprenticeship & 1 \\
\hline University qualification & 8 \\
\hline State of residence in Australia & $(n=28)$ \\
\hline VIC & 7 \\
\hline NSW & 13 \\
\hline QLD & 4 \\
\hline SA & 1 \\
\hline WA & 3 \\
\hline Number of children (parents only) & $(\mathrm{n}=19)$ \\
\hline 1 & 3 \\
\hline 2 & 5 \\
\hline 3 & 6 \\
\hline 4 & 5 \\
\hline
\end{tabular}

aSMA type 2 in the family.

bSMA type 1 in two families, SMA type 2 in one family.

CSMA type 1 in three families, SMA type 2 in one family.

dSMA type 1 in the family.

eMissing data for three participants. 
of type 3), while there were five other relatives of children with type 1 and three of type 2. Not all questions were completed by each respondent; therefore the value of $n$ varies and is noted throughout. There are an estimated 85 families who are members of the SMAAA but, based on the HREC approved protocol, the SMAAA could not disclose further details of their membership to the researchers. Therefore, we cannot determine an exact response rate, but estimated the family response rate overall as $25 / 85(29.4 \%)$.

First noticing symptoms and reported age at diagnosis of SMA Parents reported the ages at which their child received a diagnosis, which ranged as follows: SMA type 1, 7 weeks to 8 months; SMA type 2, 5-24 months; SMA type 3, 18-42 months (see Table 2). They also reported who first noticed symptoms and when, although did not specifically report the age of the child at the very first visit to a health professional. The delay between the time when symptoms were first noticed and when a diagnosis was received ranged as follows: SMA type 1, less than a week to 8 months; SMA type 2: 4-16.5 months; SMA type 3, 6-15 months (Table 2). The mean time between first noticing symptoms to receiving a diagnosis was: SMA type 1, 2.9 months; SMA type 2, 9.8 months; SMA type 3, 8.3 months.

Many parents reported having a gut feeling something was wrong. In about half of the families, the mother noticed symptoms first and in other families grandmothers and aunts were the first to notice (Table 2).

'You couldn't put your finger on it but you knew that there was something.'

P21, grandmother of girl, SMA T2
Experience raising previous children in the family made parents more alert to their concerns, especially when the child's abilities regressed:

'You indistinctly know something is wrong even though babies can develop at different rates. Having two other kids already you are probably a[t]tuned to what the developmental phases are. The fact that he had already started to try and roll, lift and support head, then started not being able to was a big clue.'

P19, father, of boy (deceased), SMA T1

All reported various emotional states upon first being aware of symptoms in their child, and these were most commonly described as feelings of denial, stress, anxiety, worry and being unsure what was happening in their child:

'I said nothing's wrong with my child, you go into denial saying 'nup, nothing's wrong, nothing's wrong."

P1, mother of girl, SMA T2

\section{Searching for answers and receiving a diagnosis of SMA in the} family

In the transition from noticing symptoms to seeking answers, participants continued to be stressed and worried, and their sense of pessimism about the future and feeling frustrated increased:

'It was really, really frustrating and scary... I just felt like... is there something wrong with me that I'm thinking there is something wrong with him, you know?'

P3, mother of boy, SMA T2

Table 2 Parent-reported details of age when symptoms were first noticed, age when receiving a diagnosis and consultations with health professionals

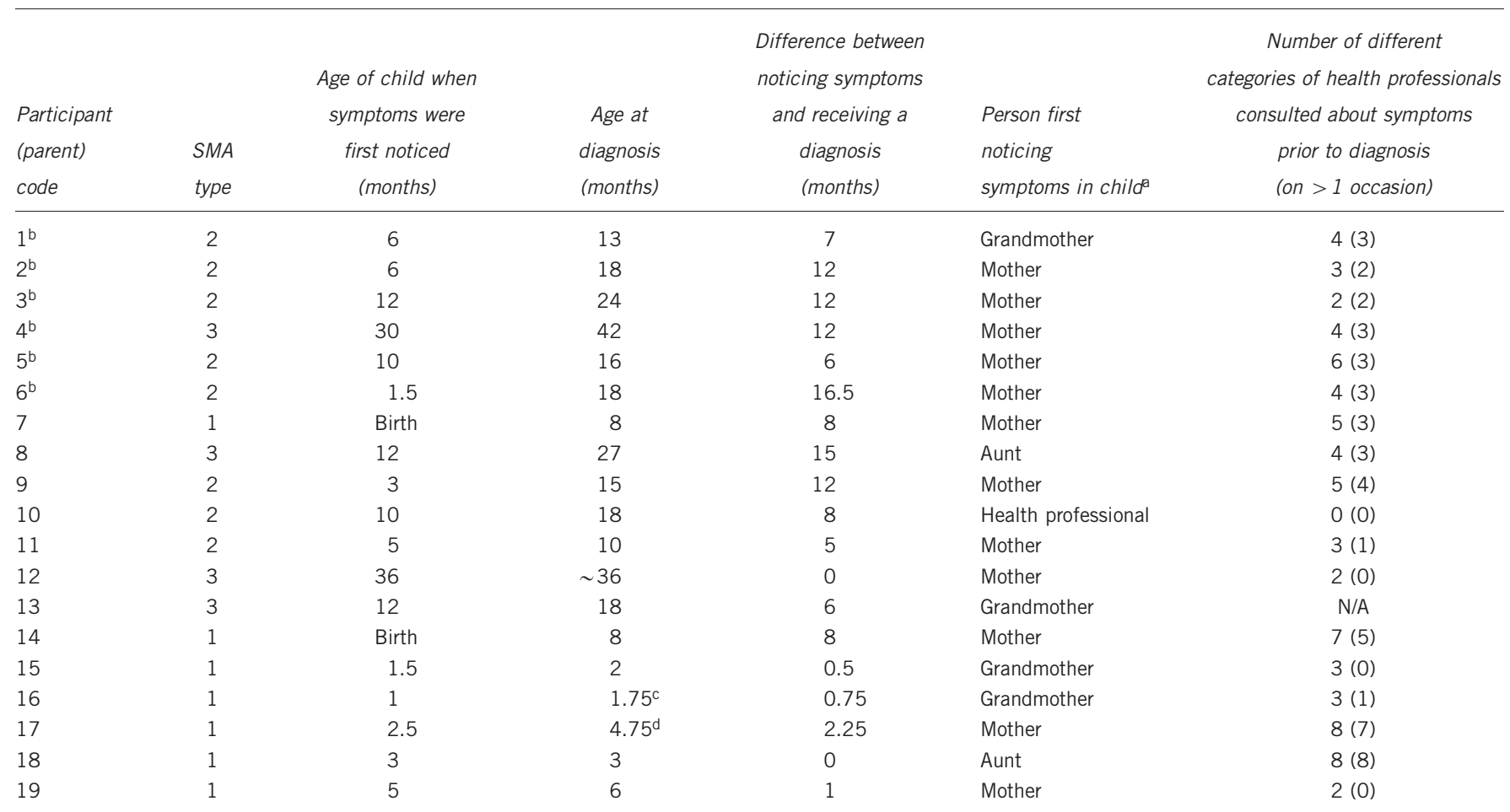

aRelationship to child.

bInformation reported in interview.

'Reported in survey as seven weeks.

dReported in survey as 19 weeks; N/A-data not available (incomplete survey) 
These feelings seemed to motivate participants to seek help from health professionals:

'In the end I just went 'nup I'm gonna get this checked... we better just in case' I said, 'just for my own peace of mind I'm having him checked."

P5, mother of boy, SMA T2

Parents consulted with various health professionals about symptoms they were noticing and throughout their journey to diagnosis. Parents reported consulting anywhere from zero (when the maternal child health nurse was the first to notice symptoms) to eight different categories of health professionals (mean number of categories $=4$ ) about symptoms they were noticing prior to receiving a diagnosis; they often visited individual health professionals several times (Table 2). These included general practitioners, maternal child health nurses, paediatricians, paediatric neurologists, naturopaths, chiropractors, dieticians, speech therapists, occupational therapists and physiotherapists. The most common health professionals consulted first were general practitioners and maternal child health nurses.

Many participants felt the process to diagnosis was a somewhat protracted journey, whereby health professionals initially tried to reassure them about the development of their child:

'I took her to about a dozen different doctors and they kept saying different things: 'she'll be fine, she's just a late developer' or 'she has an immature nervous system' which they were accounting for the tremor.'

P6, mother of girl, SMA T2

'I went to a doctor when my child was around 14 months of age stating my child is not walking and we have been doing therapy since 10 months of age. The doctor dismissed it and said his child didn't walk 'til they were two.'

P10, mother of boy, SMA T2

When health professionals decided to act, participants appeared uncertain about the process of testing and unprepared for the outcome:

'Well we didn't really know what to expect because they didn't tell us what they were testing him for, they just ordered all the tests and it was just really a waiting game.'

P2, mother of boy, SMA T2

...I think that it should be explained to the parents if their child is being tested for it, exactly what it is... I was not told and I got a big shock... he just rang up and said 'yeh she's tested positive' and sort of hung up... I had no idea what it was or what it was going to entail...'

P6, mother of girl, SMA T2

The majority of participants were given the diagnosis of SMA by neurologists and paediatricians. Participants described reacting in various ways to the diagnosis, which were mainly feelings of shock, numbness, potential rejection/denial, worry, and with increasing feelings of sadness.

'I said 'I don't want to know her, I don't want to know my daughter.... 'I can't deal with this, I can't deal with this."

P1, mother of girl, SMA T2

'I had already read about muscular dystrophy and things like that... I just knew that ok that sounds really bad, it's got a terrible name... I don't remember a lot of the stuff that he said because I was just in real shock...'

P3, mother of boy, SMA T2

Parents also commented on how doctors described SMA:

'...we were told SMA [type] 1 was when children usually die before the age of two. I began crying because our son had just passed two. The doctor continued saying SMA type 2 was when children were usually bedridden and never walk, but this was not our son. SMA type 3 included children that would walk and muscles would deteriorate over time. We were devastated.'

P8, mother of boy, SMA T3

Potentially misleading information from health professionals

In some instances, parents felt the information given was potentially misleading:

'Doctor basically told us to go home and wait for the funeral. Our daughter would probably never go to school, would have life expectancy of 5-7 years of age, would not require physio etc. Total bull.'

$$
\text { P9, mother of girl (7 years), SMA T2 }
$$

This mother's daughter was diagnosed with SMA type 1 at 8 months of age:

'In the end my child attends university and has made it to 19. It could have been so much better if they had acted as though there was some chance of survival.'

P14, mother of girl (currently 19 years), SMA T1

All participants felt that it is important to raise awareness and provide formal education to health professionals about the symptoms of SMA. Others commented on the need for written information:

'Telling our family was hard. We would have liked some information to take to our families because we got bombarded with questions that we didn't know how to answer.'

P8, mother of boy, SMA T3

\section{Reflections on timing of the diagnosis}

The majority of participants believed that the diagnosis could have been earlier; however, there were mixed opinions about whether or not an earlier diagnosis would have benefited the child:

'Nope, because there's nothing really, like there's no real medications he can go on that's going to stop it or anything...'

P4, mother of boy and girl, SMA T3

'...we just would've probably had the physios, everything would've started earlier and may be he would be able to walk a bit more without aids... we may have been able to just get him moving a bit quicker and having a bit more strength exercises....'

P5, mother of boy, SMA T2

'I am glad we didn't have the torture of knowing our daughter was going to pass away earlier. We found out one week before she passed 
away. There is nothing we could have done about it, we did all we could and let her suffer as least as possible.'

P17, mother of girl (deceased), SMA T1

Despite these contrasting views, some participants felt that an earlier diagnosis would have been beneficial for themselves:

'I think it would've given me something that I was... wanting to know earlier... I guess it would've saved me a lot of... worry and confusion.'

P3, mother of boy, SMA T2

Parents' and relatives' views on screening to diagnose SMA earlier The notion of screening children as a way of diagnosing SMA earlier was discussed with participants; prompts included population screening of newborns or in early childhood, either to the entire population or targeted based on (minimal) parental concerns (as opposed to testing based on symptoms of SMA in the child). Although many participants felt that the child could have received an earlier diagnosis, there was an obvious tension when deciding on a time point for such screening. This tension appeared to be because of the broad phenotypic nature of SMA, even within a specific type, and the difficulty of predicting the level of disability. On the other hand, carrier screening was often raised by parents in the interviews as an option without being prompted; overall, this was a more favoured approach to screening the population with all participants agreeing (except three who neither agreed nor disagreed), that it is important to offer SMA carrier screening to couples planning pregnancy; carrier screening during pregnancy was also supported but to a lesser extent. Participants also found it difficult to decide on an appropriate stage when to offer carrier screening pre-pregnancy:

'...it's hard to put an age on it... I was going to say when couples get engaged but I mean not everyone is going to do that...'

$\mathrm{P} 21$, grandmother of girl, SMA T2

\section{DISCUSSION}

This study is the first to explore families' experiences of the journey to diagnosis for SMA. The ages at diagnosis for childhood SMA are earlier than $\mathrm{DMD}^{4}$ and $\mathrm{FXS},{ }^{2}$ which presumably reflects the underlying nature of the progressive degeneration of the $\alpha$ motor neurons in the spinal cord leading to SMA. Nevertheless, we have shown that, similar to other genetic conditions presenting with developmental delay, such as DMD and FXS, there is a delay in receiving a diagnosis of SMA for children once the family has noticed symptoms in the child. There is a wide spectrum of 'normal' development at any given time point and therefore it can be difficult for health professionals to differentiate between healthy children showing transient developmental delay and developmental disability caused by SMA, or indeed other genetic conditions. Given this, health professionals may take a 'wait and see' approach before considering performing relevant tests. Although this is standard practice until a child surpasses the milestone age, this can clearly be frustrating and distressing for parents who experienced a perceived diagnostic odyssey, and who consulted with many different health professionals in their search for answers.

Participants reported the stress, anxiety, frustration and self-doubt that resulted from trying to convince health professionals that there was a problem. Once a diagnosis was made, many parents clearly struggled with the shock and the information imparted, a situation that has been described for other conditions. ${ }^{18,19}$ Our findings suggest that this initial shock may result from parents not being told about the condition/s being tested prior to testing itself, as well as the manner in which the diagnosis was disclosed. It is likely health professionals did not want to alarm parents about the possible cause of their child's problems until the diagnosis was confirmed. Delivering a diagnosis with poor prognosis is always difficult, but there has been much literature on how this can be managed better, ${ }^{19-21}$ including advice from parents themselves. ${ }^{22}$

When thinking about an earlier diagnosis for their child, our data suggest that most participants would have wanted an earlier diagnosis regardless of whether it would have benefited their child as it would have prevented worry and confusion for family members; however, their opinions on the timing of when the diagnosis could occur and how varied.

The reported delays in diagnosis of inherited conditions which present with developmental delay support the notion of considering the implementation of a systematic approach to diagnosing these conditions at a population level, rather than relying on case-finding in a clinical setting - through identifying carriers or children themselves. Population screening to diagnose such conditions could occur at an earlier time point, such as in the newborn or infant period. ${ }^{23,24}$ Newborn screening (NBS) has been widely used over many decades to identify children with various life-threatening conditions. ${ }^{25}$ There is much discussion about possible expansion and evaluation of NBS programs to include other conditions such as DMD ${ }^{25-27}$ and FXS. ${ }^{28-}$ ${ }^{30}$ Similarly, SMA could be considered for inclusion. ${ }^{7}$

When presented with screening options, such as screening children in the newborn or infant period, participants in our study highlighted the complexity of choosing an appropriate time to test, because of the variability in the phenotype between SMA types and even within the same SMA type. If screening were to be implemented, it is likely that testing would involve mutation analysis thereby identifying every type of SMA, including late childhood and adult-onset types, rather than screening based on biochemical markers or clinical features. Thus, it was difficult for participants to make a clear decision on a time point for screening of children and participants' views varied on when screening might occur. We have explored these participants' perspectives on population screening of newborns and children for SMA in greater depth and compared and contrasted these with views of parents of children with DMD and parents of healthy children in the general population; the findings from this larger study will form the basis of a separate publication.

It is interesting to note that, when asked to consider a populationbased screening approach for SMA, most participants in our study raised the idea of carrier screening as their first response, and there was overwhelming support for this to be offered to couples when planning a pregnancy, as this would provide greater reproductive options to couples. Nevertheless, despite their initial support for carrier screening, participants acknowledged the fact that it would not be easy to access eligible individuals for a carrier screening program. One pilot study in the USA addressed the clinical acceptability of carrier screening for SMA in the general population, finding that $60 \%$ of women seeking prenatal genetic counselling services accepted SMA carrier testing at the time of counselling. ${ }^{7}$ About $75 \%$ of women had not heard of SMA previously and all received pre-test genetic counselling, although their decision-making processes and their views about screening at different life stages were not explored. ${ }^{7}$ FXS and cystic fibrosis studies consistently show a preference for carrier screening over other screening options, with this occurring ideally before pregnancy. ${ }^{31-33}$ Increasingly, carrier screening for panels of 
genetic conditions are being offered ${ }^{34}$ and a carrier screening program combining testing for SMA, FXS and cystic fibrosis has recently been launched in Victoria, Australia; uptake of testing has not yet been determined. As more conditions are added to carrier screening panels, it will be important to understand couple's decision-making, especially for conditions in which prognostic outcomes are not always clear.

The views of parents and relatives in this study may reflect those of a biased sample as all participants and respondents were self-selecting and were recruited through the support group, SMAAA. We acknowledge this is a limitation of the study, as well as the low estimated family response rate. It is possible that people who are part of SMAAA are more proactive and more prepared to engage in research around this topic, and we cannot generalise our findings to all families with SMA in Australia. Furthermore, in hindsight, it might have been useful to have also interviewed parents of children with SMA type 1 as findings from the survey indicated this group also underwent some diagnostic delay.

\section{CONCLUSION}

This study suggests that receiving a diagnosis of SMA is later than optimal and families felt extremely stressed, worried and frustrated throughout the process. It makes unique contributions to knowledge regarding families' perceptions about the process of receiving a diagnosis and screening for SMA. To reduce their diagnostic journey, enable earlier access to intervention and inform reproductive planning, it is likely that multiple approaches (ie newborn or infant screening, carrier screening and raising health professional awareness) will be necessary to minimise the substantial impact on families. Further research, with SMA families and the general population will be instrumental in evaluating potential strategies.

\section{CONFLICT OF INTEREST}

The authors declare no conflict of interest.

\section{ACKNOWLEDGEMENTS}

We would like to thank the families who participated in this study, and Julie Cini from the Spinal Muscular Atrophy Association Australia for distributing invitations to families. Funding for this study was provided by the Murdoch Childrens Research Institute. This work was supported by the Victorian Government's Operational Infrastructure Support Program.

1 Bellman M, Byrne 0, Sege R: Developmental assessment of children. BMJ 2013; 346: e8687.

2 Bailey Jr DB, Raspa M, Bishop E, Holiday D: No change in the age of diagnosis for fragile $X$ syndrome: findings from a national parent survey. Pediatrics 2009; 124 527-533.

3 Firth MA: Diagnosis of Duchenne muscular dystrophy: experiences of parents of sufferers. BMJ (Clin Res Ed) 1983; 286: 700-701.

4 Bushby KM, Hill A, Steele JG: Failure of early diagnosis in symptomatic Duchenne muscular dystrophy. Lancet 1999; 353: 557-558.

5 Smith M, Calabro V, Chong B, Gardiner N, Cowie S, du Sart D: Population screening and cascade testing for carriers of SMA. Eur J Hum Genet 2007; 15: 759-766.
6 Pearn J: Incidence, prevalence, and gene frequency studies of chronic childhood spinal muscular atrophy. J Med Genet 1978; 15: 409-413.

7 Prior TW, Snyder PJ, Rink BD et al: Newborn and carrier screening for spinal muscular atrophy. Am J Med Genet A 2010; 152A: 1608-1616.

8 Pearn J: Classification of spinal muscular atrophies. Lancet 1980; 1: 919-922.

9 Lefebvre S, Burglen L, Reboullet S et al: Identification and characterization of a spinal muscular atrophy-determining gene. Cell 1995; 80: 155-165.

10 Parsons DW, McAndrew PE, Monani UR, Mendell JR, Burghes AH, Prior TW: An 11 base pair duplication in exon 6 of the SMN gene produces a type I spinal muscular atrophy (SMA) phenotype: further evidence for SMN as the primary SMA-determining gene. Hum Mol Genet 1996; 5: 1727-1732.

11 Prior TW, Swoboda KJ, Scott HD, Hejmanowski AQ: Homozygous SMN1 deletions in unaffected family members and modification of the phenotype by SMN2. Am J Med Genet A 2004; 130A: 307-310

12 Munsat TL, Davies KE: International SMA consortium meeting. (26-28 June 1992, Bonn, Germany). Neuromuscul Disord 1992; 2: 423-428.

13 Farrar MA, Vucic S, Johnston HM, du Sart D, Kiernan MC: Pathophysiological insights derived by natural history and motor function of spinal muscular atrophy. J Pediatr 2013; 162: 155-159.

14 Creswell J, Plano Clark V: Designing and Conducting Mixed Methods Research. California: Sage Publications, Inc., 2007.

15 Bluff R: Grounded theory; in: Cluett ER, Bluff R (eds). Principles and practice of research in midwifery. Edinburgh: Churchill Livingstone/Elsevier, 2006; pp 293.

16 Hsieh HF, Shannon SE: Three approaches to qualitative content analysis. Qual Health Res 2005; 15: 1277-1288.

17 Rice PL, Ezzy D: Qualitative research methods. Melbourne: Oxford University Press, 1999.

18 Green JM, Murton FE: Diagnosis of Duchenne muscular dystrophy: parents' experiences and satisfaction. Child Care Health Dev 1996; 22: 113-128.

19 Hill V, Sahhar M, Aitken M, Savarirayan R, Metcalfe S: Experiences at the time of diagnosis of parents who have a child with a bone dysplasia resulting in short stature. Am J Med Genet A 2003; 122A: 100-107.

20 Fallowfield L, Jenkins V: Communicating sad, bad, and difficult news in medicine. Lancet 2004; 363: 312-319.

21 Sparks L, Villagran MM, Parker-Raley J, Cunningham CB: A patient-centered approach to breaking bad news: communication guidelines for health care providers. J App/ Commun Res 2007; 35: 177-196.

22 Levenson D: Communication with parents counts. Am J Med Genet A 2010; 152A fmvii-fmvi.

23 Ross LF: Screening for conditions that do not meet the Wilson and Jungner criteria: the case of Duchenne muscular dystrophy. Am J Med Genet A 2006; 140: 914-922.

24 Laing NG: Multiplicity of experimental approaches to therapy for genetic muscle diseases and necessity for population screening. J Muscle Res Cell Motil 2008; 29: 247-252.

25 Burton H, Moorthie S: Expanded Newborn Screening; a Review of the Evidence. PHG Foundation, 2010.

26 Bradley DM, Parsons EP, Clarke AJ: Experience with screening newborns for Duchenne muscular dystrophy in Wales. BMJ 1993; 306: 357-360.

27 Parsons EP, Clarke AJ, Hood K, Lycett E, Bradley DM: Newborn screening for Duchenne muscular dystrophy: a psychosocial study. Arch Dis Child Fetal Neonatal Ed 2002; 86: F91-F95.

28 Bailey Jr DB: Newborn screening for fragile X syndrome. Ment Retard Dev Disabil Res Rev 2004; 10: 3-10.

29 Hill MK, Archibald AD, Cohen J, Metcalfe SA: A systematic review of population screening for fragile X syndrome. Genet Med 2010; 12: 396-410.

30 Skinner D, Choudhury S, Sideris J et al: Parents' decisions to screen newborns for FMR1 gene expansions in a pilot research project. Pediatrics 2011; 127: e1455-e1463.

31 Metcalfe S, Jacques A, Archibald A et al: A model for offering carrier screening for fragile $\mathrm{X}$ syndrome to nonpregnant women: results from a pilot study. Genet Med 2008; 10: 525-535.

32 Skinner D, Sparkman KL, Bailey Jr DB: Screening for fragile $X$ syndrome: parent attitudes and perspectives. Genet Med 2003; 5: 378-384.

33 Green JM: Principles and practicalities of carrier screening: attitudes of recent parents. J Med Genet 1992; 29: 313-319.

34 Srinivasan BS, Evans EA, Flannick J et al: A universal carrier test for the long tail of Mendelian disease. Reprod Biomed Online. 2010; 21: 537-551. 